\title{
BMJ Open Dementia prevalence in a population at high vascular risk: the Trinidad national survey of ageing and cognition
}

\author{
Gershwin Davis, ${ }^{1}$ Nelleen Baboolal, ${ }^{2}$ Amanda Mc Rae, ${ }^{3}$ Robert Stewart ${ }^{4,5}$
}

To cite: Davis G, Baboolal N, Mc Rae A, et al. Dementia prevalence in a population at high vascular risk: the Trinidad national survey of ageing and cognition. BMJ Open 2018;8:e018288. doi:10.1136/ bmjopen-2017-018288

- Prepublication history for this paper is available online. To view these files, please visit the journal online (http://dx.doi. org/10.1136/bmjopen-2017018288).

Received 26 June 2017 Revised 16 December 2017 Accepted 17 January 2018

Check for updates

${ }^{1}$ Faculty of Medical Sciences, The University of the West Indies, EWMSC, Champs Fleurs, Trinidad and Tobago

${ }^{2}$ Department of Clinical Medical Sciences, The University of the West Indies, EWMSC, Champs Fleurs, Trinidad and Tobago ${ }^{3}$ Department of Preclinical Sciences, The University of the West Indies, EWMSC, Champs Fleurs, Trinidad and Tobago

${ }^{4}$ Department of Psychological Medicine, King's College London (Institute of Psychiatry, Psychology and Neuroscience), London, UK

${ }^{5}$ South London and Maudsley NHS Foundation Trust, London, UK

Correspondence to

Dr Gershwin Davis;

gershwin.davis@sta.uwi.edu

\section{ABSTRACT}

Objective To investigate the dementia prevalence in a country with high levels of cardiovascular risk factors Design and methods Older people in Trinidad are recognised to have particularly high levels of cardiovascular risk factors. We carried out a survey in a nationally representative sample of people aged $\geq 70$ years using household enumeration. Dementia status was ascertained using standardised interviews and algorithms from the 10/66 schedule and age-specific prevalence were compared with identically defined output from the 10/66 surveys of 16536 residents in eight other lowincome and middle-income countries.

Results 0 f 1832 participants (77.0\% response rate), dementia was present in 442 (23.4\%). Prevalences were $12.0 \%$ in persons aged $70-74$ years, $23.5 \%$ at $75-79,25.8 \%$ at $80-84,41.3 \%$ at $85-89$ and $54.0 \%$ in those aged $\geq 90$ years. Prevalence ratios compared with averages from 10/66 surveys in these age groups were 2.2, 2.6, 1.6, 1.7 and 1.6, respectively, and were 2.7, 2.8, $1.7,1.4$ and 0.8 , respectively, compared with previously published consensus estimates for the Latin American region. Dementia was significantly associated with reported stroke and diabetes in logistic regression models adjusted for sociodemographic status and other vascular risk factors (OR $(95 \% \mathrm{Cl}) 4.40$ (2.70 to 7.19$)$ and 1.56 (1.20 to 2.03), respectively). Projected national numbers of people with dementia (18 206) were $70 \%-100 \%$ higher than those estimated using most recent regional consensus prevalences.

Conclusion In a nation with high levels of vascular risk, dementia prevalence is higher than expected, particularly at the lower end of the $70+$ age range. International prevalence projections may need to take into account risk status as well as age structures.

\section{INTRODUCTION}

As well as having a profound impact on individuals and those who care for them, dementia is one of the most costly conditions for national health and social care systems. ${ }^{1-3}$ With rapid demographic ageing occurring in both high-income and lower income nation settings, projected estimates of case numbers at regional and national levels are vital for policymaking, planning and allocation of health and welfare resources. ${ }^{3-7}$ These

\section{Strengths and limitations of this study}

To our knowledge, ours is one of very few studies to investigate dementia prevalence in a nationally representative sample, rather than in a defined geographic catchment.

- The restriction of sampling to residents aged $\geq 70$ (rather than $>65$ ) years increased the numbers in older age groups and thus the precision of prevalence estimates in these age ranges.

- The study used a dementia diagnostic schedule which has been previously validated in a range of low-income and middle-income countries through the $10 / 66$ programme.

- Response rates were reasonably high for a community survey; however, non-response bias needs to be considered when inferring prevalences.

- Although robustly validated and widely used, the $10 / 66$ diagnostic algorithm is probabilistic and not equivalent to a clinical interview and examination; furthermore, the version used here does not seek to determine the subtypes of dementia.

projections require good quality information on the numbers of people in given age ranges and the proportions who can be expected to have dementia. However, while population age structures can be reasonably estimated and projected from national census data, ${ }^{8-10}$ obtaining relevant age-specific dementia prevalence data is more challenging. Because large proportions of people with dementia remain undiagnosed, administrative data from healthcare cannot be relied on; instead, prevalences have to be inferred from epidemiological surveys with valid case-finding instruments, and because it is beyond the resources of most authorities to commission dedicated surveys, international consensus estimates have to be used. ${ }^{11}$ One drawback with this approach is that, apart from broad demographic profiles, it does not take into account risk factors for dementia which may vary between populations and might influence prevalence. In particular, cardiovascular risk factors such as hypertension and diabetes 
are well recognised to be associated with a higher risk of dementia at an individual level, ${ }^{12-15}$ but their impact on national prevalence is not known. This is important because there is substantial national and regional variation in vascular risk factors ${ }^{16}$ and in cardiovascular outcomes, such as myocardial infarction and stroke; therefore, it is problematic for a nation with high levels of vascular risk factors to be assuming dementia prevalences calculated in low-risk settings. In addition, cardiovascular risk profiles have been changing rapidly in many nations; these might well influence future dementia prevalence but the impact cannot currently be estimated.

Attempts to compare dementia prevalence between nations with different vascular risk profiles has been limited by inconsistencies in sampling and, particularly, outcome measurement. The $10 / 66$ consortium made substantial advances through the development of a single dementia assessment protocol with established cross-cultural validity, ${ }^{17-19}$ although its surveys to date have been limited to regional catchment samples rather than nationally representative populations. In order to investigate this issue further, we applied 10/66 measurement protocols in a recently conducted national survey of dementia prevalence in Trinidad and Tobago, a country recognised to have high cardiovascular risk indices: ranked 4th highest for diabetes mortality, 14th for hypertension mortality and 42nd for coronary heart disease mortality. ${ }^{20}$ In addition to providing relevant information for the government, health and welfare agencies to plan adequate services for current and future cases of dementia, and to begin identifying and evaluating specific risk factors, we sought to test the hypothesis that this country with a recognised high level of vascular risk ${ }^{21}$ would also have a higher than expected prevalence of dementia.

\section{METHODS}

\section{Study design and sampling}

A comprehensive cross-sectional survey was carried out of all residents aged $\geq 70$ years living in randomly selected electoral enumeration districts (EDs) in Trinidad. The objective was to attain a nationally representative sample from this island; resources were not sufficient at the time to sample the smaller population of Tobago. Administrative units in Trinidad comprise 14 municipalities and 2664 EDs. A municipality is defined as a primarily urban political unit, having corporate status and powers of self-government, and an ED is a geographical area comprising approximately 150-200 households. For the purpose of this survey, 120 EDs were selected using stratified random sampling with proportional allocation to each municipality stratum. The numbers of EDs allocated to each municipality were thus proportional to the population size of the particular municipality. Sample size calculations indicated that this would be sufficient to recruit an overall sample of 2000 , which would allow estimation of a typical dementia prevalence of $4.5 \%$ with a precision of $\pm 0.9 \%$.
There were two phases to the survey. In phase I, household enumeration was conducted in the 120 randomly selected EDs, with maps provided by the Central Statistical Office to assist in the exercise. In each ED, all households were approached, field workers went door to door and inquired about individuals living in the household who were $\geq 70$ years. In phase II, field workers revisited the specific households, that is the households with at least one resident aged $\geq 70$ years, as identified in phase I door knocking phase, to interview index participants, informants (someone who knew a participant well) and heads of household. All enumerated individuals resident in the 120 EDs and aged $\geq 70$ years were invited to participate. During phase I, the enumerators were advised that data collection in two of the selected EDs would be too risky because of recent crime and violence levels; therefore, two other EDs were randomly selected to replace these. At least one appropriate informant was found for all participants in the study and there were no participants for whom an informant could not be found. All participants gave informed consent or, if capacity to provide consent was lacking, this was provided by an appropriate proxy.

\section{Interviews}

The study team consisted of a project coordinator, six supervisors and 30 field enumerators/interviewers. The latter had previously carried out enumerations and interviews on the national census and a number of other national surveys for the Central Statistical Office. All staff were trained on the instrument by one of us RS who has participated in 10/66 Consortium instrument development and research, and training covered the sampling protocol and procedures, as well as structured interviewing techniques and the specific instruments. Field interviewers were regularly checked and supervised and reported to the supervisors during the study, although there was no formal inter-rater agreement testing in this survey. Interviews were generally carried out in participants' homes and all participants received the full assessment.

\section{Measurements}

The study used the 10/66 short dementia diagnostic schedule and accompanying algorithm. This comprises the following instruments which were administered to all participants:

1. The Community Screening Instrument for Dementia; this has been widely used in international and cross-sectional research, for example, as a component of the 10/66 schedule and in the earlier IndianapolisIbadan studies. The instrument comprises both an education-fair cognitive assessment and a fully structured informant interview.

2. The CERAD 10-word list learning task. An additional component of the 10/66 schedule, the task presents the word list three successive times for immediate recall, followed by a single delayed recall test after a distraction task. 
3. The Euro-D scale: a fully-structured 12-item questionnaire enquiring about common depressive symptoms. It was originally developed as a means of developing a harmonised depression instrument for the EURODEP surveys of late-life mental health, where different schedules had been used, but has since been widely applied and evaluated.

The 10/66 short dementia diagnostic algorithm was applied to output from these instruments in order to ascertain presence or absence of dementia as a binary variable. The 10/66 short schedule is identical to the original 10/66 schedule ${ }^{22}$ in its inclusion of the Community Screening Instrument for Dementia and CERAD 10-word list recall task, but substitutes the Euro-D depression instrument for the longer Geriatric Mental State (of which the Euro-D is a subcomponent). As recently published ${ }^{19}$, the $10 / 66$ short schedule retains high sensitivity and specificity for dementia ascertainment (on data from 25 international centres) and was comparable to the longer instrument in dementia prevalence estimation across previous 10/66 surveys (on data from 16536 participants in 12 sites from eight countries). All components of the algorithm and the source instruments have been published in an open access source. ${ }^{19}$

In addition to dementia status, information was obtained on the following demographic factors: age, gender, level of education (none, primary, secondary and tertiary), ethnicity (African, East Indian and mixed/other) and previous main occupation (agricultural—self-employed, agricultural— other, semi-skilled, skilled, unskilled, clerical/professional and other). A questionnaire on presence or not of the following health conditions was administered, with corroboration sought where possible from the informant: angina, heart disease, stroke, diabetes, high cholesterol and hypertension.

\section{Statistical analysis}

Because of the proportional sampling, unweighted analyses were carried out; in addition, because the large majority of participants $(83.6 \%)$ were recruited from single households, it was not felt necessary to adjust for within-household clustering. Prevalences of dementia were described according to the listed covariates and ORs calculated to describe these associations, followed by logistic regression modelling and testing of the associations between dementia and reported health conditions. We fitted a series of logistic regression models to assess the individual and joint effects of various health indicators on dementia, adjusting for demographic characteristics. In the first set of models, we fitted six separate logistic models with indicators for either angina, heart disease, stroke, diabetes, high cholesterol or hypertension as the primary predictors of interest, adjusting for age, sex and ethnicity. In the second set of models, we fitted six similar logistic models, but additionally adjusted for education and previous occupation. Finally, in a further model, we included all six health indicators and demographic covariates to assess their joint effects on dementia. Five age groups were used for all prevalence analyses: 70-74, $75-79,80-84,85-89$ and $90+$ years. CIs for age-group-specific prevalences were provided, taking into account within-household clustering, but clustering adjustments were not employed in other analyses.

In order to contextualise prevalences in Trinidad, the $10 / 66$ short-schedule algorithm was run, with permission, on data from previous $10 / 66$ surveys. ${ }^{22}$ Findings from these surveys have been published previously, ${ }^{23}$ and data were available on 16536 participants in 12 sites from eight countries: Cuba, Dominican Republic, Peru, Venezuela, Mexico, India, China and Puerto Rico. Prevalences from identical schedules could thus be compared across harmonised age groups. Having graphically displayed these comparisons, we calculated prevalence ratios within each age group with $95 \%$ CIs, comparing Trinidad findings with pooled estimates from the 10/66 sites. For illustrative purposes, we also described ratios comparing Trinidad findings with published consensus estimates from Latin America and USA/Canada.

We sought to estimate numbers of people with dementia in Trinidad and Tobago, in order to provide this information for national policy-makers. For the 70+ year age range sampled in the survey, we used national 2011 census numbers for men and women in the 5 year age groups defined for the survey, multiplied these by observed age-group-specific prevalences, and summed the results. We then supplemented this total with estimated numbers for the 60-69year age group taking 2011 census population sizes for 60-64 and 65-69year age groups and multiplying these by assumed prevalences of $1.3 \%$ and $2.4 \%$, respectively-the most recent consensus prevalences for Latin America, ${ }^{24}$ and thus judged to be the most appropriate estimates to use. The total number of people with dementia calculated using the survey data was then compared with hypothetical projections using consensus prevalences from Latin America and North America $^{24}$ applied to the same census strata.

\section{RESULTS}

Of the 2378 individuals enumerated and approached, $1898(79.8 \%)$ participated. Of these, sufficient data were available on $1832(96.5 \%), 442$ of whom were classified as having dementia, giving an overall prevalence of $24.1 \%$ (95\% CI 22.1 to 26.1 ) in the $70+$ yearage range. The basic demographic characteristics of the interviewed sample were similar to the broader population of individuals aged $\geq 70$ years in Trinidad and Tobago. The percentages of man and woman in the study were the same as census data with $37 \%$ of African and East Indian ethnicity compared with $34 \%$ and $35 \%$, respectively, from census data. Concerning the EDs, mean numbers of participants per ED were 18.0 (SD 9.3; range 1-45). Prevalences by age and gender are displayed in figure 1 , and associations between covariates and dementia are summarised in table 1. Considering demographic factors, 
80

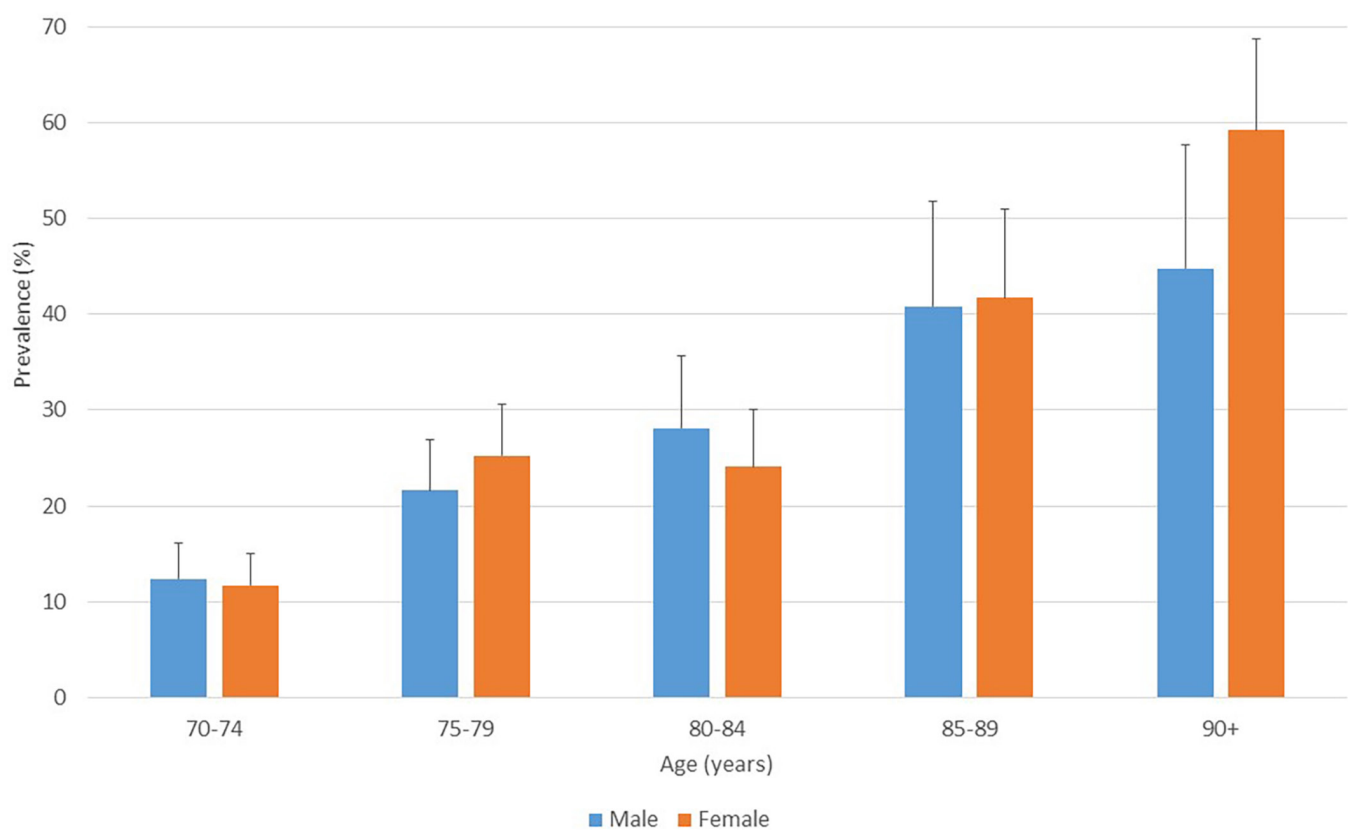

Figure 1 Dementia prevalence (\%) by age and gender (with upper $95 \% \mathrm{Cl}$ ).

the prevalence of dementia was associated with increased age and lower education, and heterogeneity between ethnic groups (lowest in the 'other' category), and by previous/current occupation (highest in self-employed agricultural workers, lowest in those reporting clerical or professional occupations). No significant gender difference was found. But considering health conditions, dementia prevalence was higher only in people for whom heart disease, stroke or diabetes was reported, and was not with angina, high cholesterol or hypertension.

In logistic regression models (table 2), the associations with stroke and diabetes remained significant and relatively unaltered in strength following adjustment of demographic and other health factors.

Prevalences from the survey are further compared in figure 2 with those from 10/66 sites for identical age groups and having applied an identically derived outcome definition. Sample sizes for 10/66 surveys ranged from 546 (rural Peru) to 2885 (Cuba) with a mean of 1383 per site (16593 total). Dementia prevalence in Trinidad was observed to be higher in all age groups than those in all/most 10/66 sites, the only exception being similar or slightly higher prevalences in the $90+$ years age range in urban and rural Mexico. Prevalence ratios by age group, comparing Trinidad with combined data for the 10/66 sites, were as follows: 2.44 (95\% CI 1.92 to 3.10$)$ at $70-74$ years; $2.76(2.27-3.36)$ at $75-79$ years; $1.61(1.31-1.98)$ at $80-84$ years; $1.70(1.39-2.08)$ at $85-89$ years; and 1.58 $(1.30-1.91)$ at $90+$ years. Comparing Trinidad dementia prevalences with published international consensus prevalences (24) in the same age groups, ratios were 2.7, 2.8, 1.7, 1.4 and 0.8 , respectively compared with the Latin
America region, and 3.5, 3.7, 2.2, 1.9 and 1.1, respectively compared with the USA/Canada region.

We multiplied our survey prevalences with census-derived national numbers in the specified age bands, and used international consensus prevalences to estimate additional numbers in 60-64 and 65-69year bands, as previously described. Through this process we estimated that there were 18206 people with dementia in Trinidad and Tobago: $10.3 \%$ of the 176833 people aged $60+$ years in the 2011 census. Hypothetical application of consensus prevalences from Latin America resulted in a projected 10535 cases, $42 \%$ lower than our estimate, and consensus prevalences from North America projected a total number of 8748 cases ( $52 \%$ lower).

\section{DISCUSSION}

In a nation recognised to have high levels of cardiovascular risk, we found high dementia prevalence in a national sample of people aged $\geq 70$ years. Coupled with the fact that stroke and diabetes were significant risk factors, our observations are consistent with an effect of risk factor status on age-specific prevalence of dementia. Projected national numbers of people affected by dementia were substantially higher than would have been estimated from international consensus data.

To our knowledge, ours is one of very few surveys ${ }^{25} 26$ of dementia prevalence in a national rather than regional survey. The Republic of Trinidad and Tobago is a twin-island state in the Caribbean, located off the east coast of Venezuela and spanning an area of approximately $5128 \mathrm{~km}^{2}$. The most recent (2011) census estimated the population to be 1328019 of whom 1267145 (95\%) 
Table 1 Sample description and factors associated with dementia prevalence

\begin{tabular}{|c|c|c|c|c|c|}
\hline Exposure & Category & Number & $\begin{array}{l}\text { Number (\%) } \\
\text { with dementia }\end{array}$ & $\chi^{2}$ (df), P value & OR $(95 \% \mathrm{Cl})$ \\
\hline \multirow[t]{5}{*}{ Age (year) } & $70-74$ & 676 & $81(12.0)$ & & Reference \\
\hline & $75-79$ & 481 & $113(23.5)$ & & 2.26 (1.65 to 3.09$)$ \\
\hline & $80-84$ & 330 & 85 (25.8) & $163.39(4),<0.001$ & 2.55 (1.82 to 3.58$)$ \\
\hline & $85-89$ & 184 & $76(41.3)$ & & 5.17 (3.56 to 7.52$)$ \\
\hline & $90+$ & 161 & $87(54.0)$ & & 8.64 (5.86 to 12.7$)$ \\
\hline \multirow[t]{2}{*}{ Gender } & Male & 806 & $183(22.7)$ & & Reference \\
\hline & Female & 1025 & $259(25.3)$ & $1.62(1), 0.203$ & 1.15 (0.93 to 1.43$)$ \\
\hline \multirow[t]{4}{*}{ Education } & None & 139 & $67(48.2)$ & & Reference \\
\hline & Primary & 1231 & $315(25.6)$ & $81.8(3),<0.001$ & 0.37 (0.26 to 0.53$)$ \\
\hline & Secondary & 253 & $30(11.9)$ & & 0.15 (0.09 to 0.24$)$ \\
\hline & Tertiary & 124 & $11(8.9)$ & & $0.11(0.05$ to 0.21$)$ \\
\hline \multirow[t]{4}{*}{ Ethnicity } & African & 686 & $167(24.3)$ & & Reference \\
\hline & E Indian & 686 & $184(26.8)$ & $9.04(3), 0.029$ & $1.14(0.89$ to 1.45$)$ \\
\hline & Mixed & 412 & $85(20.6)$ & & $0.81(0.60$ to 1.09$)$ \\
\hline & Other & 48 & $6(12.5)$ & & $0.44(0.19$ to 1.06$)$ \\
\hline \multirow[t]{7}{*}{ Occupation } & Unskilled & 273 & $58(21.2)$ & & Reference \\
\hline & Semiskilled & 145 & $35(24.1)$ & & $1.18(0.73$ to 1.90$)$ \\
\hline & Skilled & 270 & $65(24.1)$ & & 1.18 (0.79 to 1.76$)$ \\
\hline & Clerical/professional & 423 & $63(14.9)$ & $40.2(6),<0.001$ & $0.65(0.44$ to 0.96$)$ \\
\hline & Other & 459 & $134(29.2)$ & & 1.53 (1.07 to 2.18$)$ \\
\hline & Agricultural-self-employed & 113 & $41(36.3)$ & & $2.11(1.31$ to 3.41$)$ \\
\hline & Agricultural- employed & 149 & $46(30.9)$ & & $1.66(1.05$ to 2.60$)$ \\
\hline \multirow[t]{2}{*}{ Angina } & Absent & 1812 & $435(24.0)$ & & Reference \\
\hline & Present & 20 & $7(35.0)$ & $1.31(1), 0.253$ & $1.71(0.68$ to 4.30$)$ \\
\hline \multirow[t]{2}{*}{ Heart disease } & Absent & 1643 & $383(23.3)$ & & Reference \\
\hline & Present & 189 & 59 (31.2) & $5.79(1), 0.016$ & 1.49 (1.08 to 2.07$)$ \\
\hline \multirow[t]{2}{*}{ Stroke } & Absent & 1752 & $400(22.8)$ & & Reference \\
\hline & Present & 80 & $42(52.5)$ & $36.8(1),<0.001$ & $3.74(2.38$ to 5.88$)$ \\
\hline \multirow[t]{2}{*}{ Diabetes } & Absent & 1250 & $281(22.5)$ & & Reference \\
\hline & Present & 582 & $161(27.7)$ & $5.83(1), 0.016$ & $1.32(1.05$ to 1.65$)$ \\
\hline \multirow[t]{2}{*}{ High cholesterol } & Absent & 1604 & $392(24.4)$ & & Reference \\
\hline & Present & 228 & $50(21.9)$ & $0.69(1), 0.407$ & $0.87(0.62$ to 1.21$)$ \\
\hline \multirow[t]{2}{*}{ Hypertension } & Absent & 997 & $248(24.9)$ & & Reference \\
\hline & Present & 835 & 194 (23.2) & $0.67(1), 0.414$ & 0.91 (0.74 to 1.13$)$ \\
\hline
\end{tabular}

were resident in the island of Trinidad (the remainder in Tobago), with an ethnic composition of $35 \%$ East Indian and 34\% African; the remainder being of European, Chinese and mixed descent. Religious denominations are similarly mixed including Roman Catholic, Hindu, Anglican, Presbyterian and Islam. Trinidad and Tobago has an ageing population, with $12 \%$ over the age of 60 years, compared with 5\% in Africa and $17 \%$ in North America. ${ }^{27}$

High levels of cardiovascular risk factors are recognised at a national level. In terms of coronary heart disease mortality, Trinidad and Tobago is ranked 42nd out of 172 countries with 134.5 deaths per 100000 per year: higher than Venezuela at 99.9, Jamaica at 77.2 and Barbados at 53.3 (20). The prevalence of diabetes in Trinidad and Tobago is one of the highest in the Americas region being $14.5 \%$ compared with $11.3 \%$ for Jamaica and $11.1 \%$ for Venezuela ${ }^{28}$ According to 2014 WHO data, diabetes mellitus mortality in Trinidad and Tobago accounted for $17.1 \%$ of total deaths, and the age-adjusted mortality rate was 128.4 per 100000 per year, ranked fourth highest in the world. Hypertension mortality accounted for $4.9 \%$ of total deaths, and the age-adjusted mortality of 36.8 per 100000 per year was ranked 14th in the world. The 2012 
Table 2 Adjusted associations of reported health conditions and dementia

\section{OR $(95 \% \mathrm{Cl})$}

\begin{tabular}{llll}
\hline & $\begin{array}{l}\text { Individually entered, adjusted } \\
\text { for age, sex and ethnicity }\end{array}$ & $\begin{array}{l}\text { Individually entered, adjusted } \\
\text { for age, sex, ethnicity, education } \\
\text { and previous occupation }\end{array}$ & $\begin{array}{l}\text { Simultaneously entered along } \\
\text { with model } 2 \text { covariates }\end{array}$ \\
\hline Angina & $1.46(0.54$ to 3.93$)$ & $1.52(0.56$ to 4.14$)$ & $1.19(0.42$ to 3.38$)$ \\
Heart disease & $1.29(0.91$ to 1.84$)$ & $1.22(0.85$ to 1.75$)$ & $1.21(0.83$ to 1.77$)$ \\
Stroke & $4.63(2.87$ to 7.48$)$ & $4.36(2.68$ to 7.09$)$ & $4.40(2.70$ to 7.19$)$ \\
Diabetes & $1.52(1.19$ to 1.93$)$ & $1.52(1.19$ to 1.94$)$ & $1.56(1.20$ to 2.03$)$ \\
\hline High cholesterol & $1.02(0.71$ to 1.45$)$ & $1.05(0.73$ to 1.49$)$ & $0.91(0.62$ to 1.33$)$ \\
Hypertension & $1.02(0.81$ to 1.28$)$ & $1.00(0.79$ to 1.26$)$ & $0.85(0.66$ to 1.09$)$ \\
\hline
\end{tabular}

Wald test. ${ }^{39}$

Pan-American STEPs chronic non-communicable disease risk factor survey in Trinidad and Tobago reported that $50 \%$ of individuals had a total cholesterol $>190 \mathrm{mg} / \mathrm{dL}$ or were on medication for raised cholesterol, $11 \%$ were diagnosed with raised blood pressure within the last month of the survey, $30 \%$ were overweight and $34 \%$ of men smoked. ${ }^{21}$ In addition, considering smoking, obesity, hypertension, poor diet and low exercise, the survey found that $65 \%$ of residents aged $45-64$ years had at least three of these five risk factors. ${ }^{21}$

Dementia prevalence has been well studied in the economically developed world ${ }^{929}$ but there have been fewer estimates from economically developing countries and, to our knowledge, all of these have been from geographic catchments rather than national samples. Population ageing is well recognised on a global scale, with particularly rapid expansions in the oldest age ranges in many lower income settings. It is assumed that there will be a comparable increase in the prevalence of chronic age-associated diseases including dementia; however, prevalence projections have largely been calculated based on projected changes in population age structures and have assumed universal and unchanging global/regional age-specific prevalence without taking into account potential changes in risk status. Cardiovascular risk factors such as hypertension, hypercholesterolaemia and diabetes are well-established risk factors for dementia. These vary internationally and are increasing in many settings; however, the potential impact on national dementia prevalence has not been empirically investigated. Our results reinforce the necessity of local prevalence studies and highlight the risks of extrapolations from pooled regional estimates.

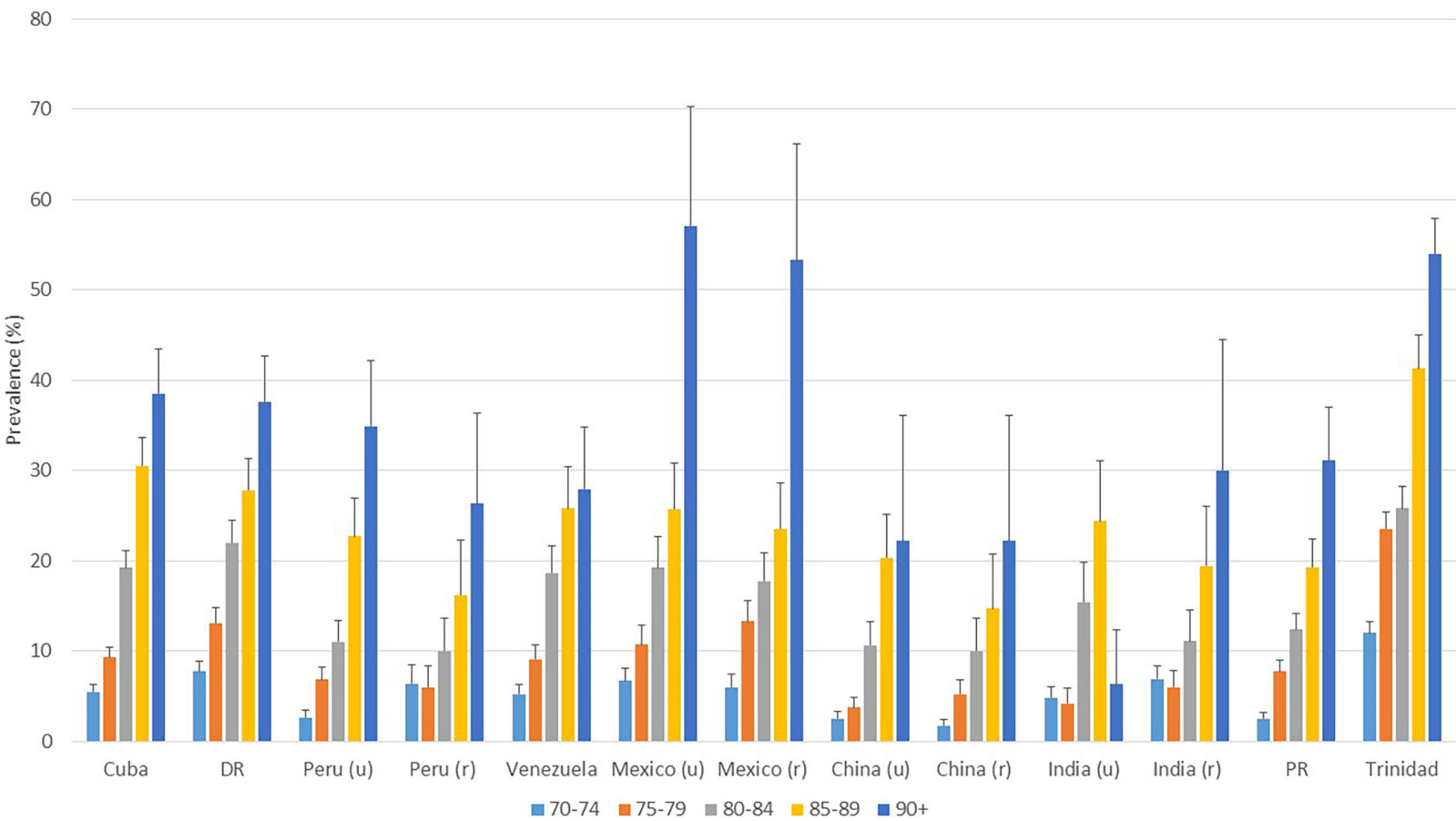

Figure 2 Prevalences of dementia (\%; SE bars) by age group across 10/66 consortium survey sites and the Trinidad survey derived using an identical ('short 10/66') case-ascertainment schedule. 
The prevalence of dementia in persons aged 70-89 in Trinidad was particularly high: 1.5-3 times higher than estimates both from 10/66 surveys and consensus predictions. An advantage of the use of the 10/66 dementia diagnostic algorithm in our survey was that it allowed direct comparisons to be made with other $10 / 66$ sites through applying an identical algorithm to identical instruments administered. Clearly, however, there are additional issues to consider before drawing conclusions. Regarding sampling methodology, both the Trinidad survey and all 10/66 surveys used complete enumeration of defined populations, the only difference being that these were a nationally representative random sample of districts in Trinidad, while 10/66 sites were specific geographic catchments (chosen to avoid unrepresentative communities but not assumed to be fully nationally representative). Considering non-response bias, participation rates were not dissimilar in the Trinidad survey compared with $10 / 66$ sites (which ranged from $72 \%$ to $98 \%$ ), ${ }^{23}$ so this would only influence prevalence differences if non-responders had a different profile in Trinidad compared with the other surveys; this seems an unlikely scenario although data were not available on people who declined to participate. Survival bias is a consideration; however, this would only give rise to the observed higher prevalences of dementia in Trinidad if people with dementia had substantially lower mortality than people with dementia in other sites, which again seems unlikely given the high levels of vascular risk recognised at a national level.

The relatively high prevalence of dementia in Trinidad is consistent with an effect of national-level vascular risk, although clearly this conclusion can only be tentative. The prevalence in the $90+$ year age group was more similar to internationally reported rates. One possible explanation is that this might reflect age-specific differences in risk effects: for example, a stronger influence of vascular risk factors in the younger age groups and a diminishing influence in those who survive longer. Raised cholesterol, either alone $e^{30}$ or combined with smoking diabetes and hypertension at midlife, ${ }^{31}$ is associated with increased risk of dementia as long as three decades later; however, cross-sectional studies have shown mixed relationships between higher blood pressure and cognition, with either no correlation or J-shaped or U-shaped associations, ${ }^{32}$ and some studies have found that hypertension in late life is associated with reduced cognitive decline. ${ }^{33}{ }^{34}$ Differences in case mortality in dementia would also have an influence on observed international differences in prevalence, although higher case mortality would obscure rather than exaggerate the higher prevalence observed in Trinidad.

In part because of the regional variability of risk factors, the WHO in 2012 encouraged all nations to conduct prevalence of dementia investigations and to establish national dementia plans. ${ }^{35}$ For example, a study in Venezuela showed that conducting nationwide prevalence studies of dementia can provide a better picture of the impact of dementia, demonstrating that the use of international estimates to predict dementia prevalence may underestimate true numbers. In particular, the prevalence of dementia in elderly people from the Caribbean coast of Venezuela was much higher than the frequencies previously reported for developing countries. ${ }^{16}$ Alzheimer's Disease International estimated that in 2010 the number of people with dementia in Trinidad and Tobago was $9000^{36}$; however, our prevalence estimates applied to 2011 national census data suggest a figure around twice as high as this, and also substantially higher than might have been estimated from regional consensus prevalences. This in turn is likely to be an underestimate, since downward-projected prevalences in the 60-64 and 65-69year age groups are likely to be higher than the $1.3 \%$ and $2.4 \%$ estimates used, although this requires further empirical research. Also the impact of participation rate need to be taken into account. It has been reported ${ }^{37}$ that population-based studies are likely to underestimate the prevalence of dementia and mild cognitive impairment especially if the participation rate is low.

A limitation in our projections was that we were not able to survey the island of Tobago; however, Tobago residents only comprise $5 \%$ of the national population and prevalences would have to differ very substantially to have a meaningful effect on estimated national numbers of people affected.

Considering strengths and limitations of the study, a key problem with many early surveys of dementia prevalence was the heterogeneity of assessments, rendering it difficult to compare between sites. The 10/66 dementia research programme was set up to address this by developing a standardised interview protocol and predictive algorithm which was valid in populations with relatively low levels of education and across cultures. For our prevalence study in Trinidad, we applied these standards, using an established diagnostic schedule and algorithm from the 10/66 programme that has been previously validated across multiple cultures and nations. ${ }^{38}$ We were also able to apply the $10 / 66$ design in surveying a national population (rather than a geographic catchment) for the first time. Our adoption of a 70+ year age cut-off clearly limits our ability to infer dementia prevalences in younger age groups; however, it was felt to be a more cost-effective means to estimate national numbers, since most people with dementia are in the upper end of the age distribution, and it allowed us to obtain reasonably precise estimates for the oldest old (eg, our sample contained 161 people aged 90+ years compared with the range of 9-96 for this age group for the listed 10/66 sites). One of the limitations of the study is that we were not able to collect blood samples or measure other markers of health status, such as blood pressure, body height and body weight. In addition, it is important to bear in mind that dementia was ascertained by a probabilistic algorithm rather than a clinical diagnosis. This approach allows for robust comparisons to be made between sites without concerns about between-clinician variability in diagnostic practice, and the algorithm itself has been evaluated against clinical diagnoses in 25 international centres ${ }^{17}$; however, precise equivalence 
between algorithm-derived and clinical diagnoses cannot be assumed. Furthermore, the algorithm applied did not allow us to define or assess dementia subtypes.

Mechanisms underlying the high prevalence of dementia observed in Trinidad clearly require elucidation in more tailored research designs, and at the national level, any link between vascular risk and dementia prevalence requires confirmation in other settings. Specifically, studies need to be done to find out whether a country's 'risk status' is associated with dementia prevalence. There is however evidence of changes over time in age-specific dementia incidence rates reported from developed nation populations, which have been proposed as due to underlying generational changes in 'brain health'. ${ }^{29}$ Our study, the first of its kind in Trinidad, seeks to support more tailored policy and better planning of services for a rapidly expanding older population, and we hope that the methodology can be readily transferred for wider adoption of national information gathering in the Caribbean region and elsewhere, as well as assisting in promoting dementia as a national priority.

Acknowledgements The authors express their thanks to the Faculty of Medical Sciences The University of the West Indies, St. Augustine for their support throughout the study We thank the Central Statistical Office (CSO) of Trinidad and Tobago and also the field workers, participants and members of households who agreed to be part of this study. This paper includes a secondary analysis of data collected by the 10/66 Dementia Research Group (www.alz.co.uk/1066). The 10/66 DRG is led by Martin Prince from Institute of Psychiatry, Psychology and Neuroscience, King's College London. The other principal investigators, data custodians and responsible for research governance in each site are Juan Llibre Rodriguez (Cuba), Daisy Acosta (Dominican Republic), Mariella Guerra (Peru), Aquiles Salas (Venezuela), Ana Luisa Sosa (Mexico), KS Jacob (Vellore, India), Joseph D Williams (Chennai, India), Ivonne Jimenez (Puerto Rico) and Yueqin Huang (China).

Contributors GD and NB: supervised/ trained field workers with respect to interview technique. AM: monitored field work execution by field work supervisors, reviewed completed questionnaires. RS: trained field workers on the 10/66 instrument and processed the algorithm. All authors: contributed to writing and interpretation of the results.

Funding This study was supported by the UWI-Trinidad and Tobago Research Development and Impact Fund (RDI) and the Ministry of Health of the Republic of Trinidad and Tobago. RS is part-funded by the National Institute for Health Research (NIHR) Biomedical Research Centre at South London and Maudsley NHS Foundation Trust and King's College London. The 10/66 Dementia Research Group's research has been funded by the Wellcome Trust Health Consequences of Population Change Programme (GR066133: prevalence phase in Cuba and Brazil; GR080002: incidence phase in Peru, Mexico, Argentina, Cuba, Dominican Republic, Venezuela and China), the World Health Organization (India, Dominican Republic and China), the US Alzheimer's Association (IIRG-04-1286: Peru, Mexico and Argentina), the Puerto Rico State Legislature (Puerto Rico) and FONACIT/ CDCH/ UCV (Venezuela).

Competing interests None declared.

Patient consent Obtained.

Ethics approval The Ethics Committee of The University of the West Indies.

Provenance and peer review Not commissioned; externally peer reviewed.

Data sharing statement No additional unpublished prevalence data from the study is available.

Open Access This is an Open Access article distributed in accordance with the Creative Commons Attribution Non Commercial (CC BY-NC 4.0) license, which permits others to distribute, remix, adapt, build upon this work non-commercially, and license their derivative works on different terms, provided the original work is properly cited and the use is non-commercial. See: http://creativecommons.org/ licenses/by-nc/4.0/ (c) Article author(s) (or their employer(s) unless otherwise stated in the text of the article) 2018. All rights reserved. No commercial use is permitted unless otherwise expressly granted.

\section{REFERENCES}

1. Alzheimer's Disease International. World Alzheimer Report 2010: The Global economic impact of Dementia, 2010.

2. World Alzheimer Report 2013. An analysis of long-term care for dementia. https://www.alz.co.uk/research/WorldAlzheimerReport 2013.pdf

3. World Alzheimer Report 2015. The Global Impact of Dementia An analysis of prevalence, incidence, cost and trends. https://www.alz .co.uk/research/WorldAlzheimerReport2015.pdf

4. United Nations, Department of Economic and Social Affairs, Population Division (2015). World Population Ageing, 2015. (ST/ESA SER.A/390).

5. Prince M, Knapp M, Guerchet M, et al. Dementia UK: update. London: Alzheimer's Society, 2014.

6. Dementia: a public health priority 2012. World Health Organization. 2012 http://www.who.int/mental_health/publications/dementia_ report_2012/en/.

7. Wu YT, Fratiglioni L, Matthews FE, et al. Dementia in western Europe: epidemiological evidence and implications for policy making. Lancet Neurol 2016;15:116-24

8. Hebert LE, Weuve J, Scherr PA, et al. Alzheimer disease in the United States (2010-2050) estimated using the 2010 census. Neurology 2013;80:1778-83.

9. Plassman BL, Langa KM, Fisher GG, et al. Prevalence of dementia in the United States: the aging, demographics, and memory study. Neuroepidemiology 2007;29:125-32.

10. Ferri CP, Prince M, Brayne C, et al. Global prevalence of dementia: a Delphi consensus study. Lancet 2005;366:2112-7.

11. Launer LJ. Counting dementia: there is no one 'best' way. Alzheimers Dement 2011;7:10-14.

12. Kalaria RN. The role of cerebral ischemia in Alzheimer's disease. Neurobiol Aging 2000;21:321-30.

13. Skoog I, Gustafson D. Hypertension, hypertension-clustering factors and Alzheimer's disease. Neurol Res 2003;25:675-80.

14. Kalaria RN, Maestre GE, Arizaga R, et al. Alzheimer's disease and vascular dementia in developing countries: prevalence, management, and risk factors. Lancet Neurol 2008;7:812-26.

15. Rizzi L, Rosset I, Roriz-Cruz M. Global epidemiology of dementia: Alzheimer's and vascular types. Biomed Res Int 2014;25:1-8.

16. Molero AE, Pino-Ramírez G, Maestre GE. High prevalence of dementia in a Caribbean population. Neuroepidemiology 2007;29:107-12.

17. Prince M, Acosta D, Chiu H, et al. Dementia diagnosis in developing countries: a cross-cultural validation study. Lancet 2003;361:909-17.

18. Prince M, Bryce R, Albanese E, et al. The global prevalence of dementia: A systematic review and metaanalysis. Alzheimer's \& Dementia 2013;9:63-75.

19. Stewart R, Guerchet M, Prince M. Development of a brief assessment and algorithm for ascertaining dementia in low-income and middle-income countries: the 10/66 short dementia diagnostic schedule. BMJ Open 2016;6:e010712.

20. World Life Expectancy 2017. http://www.worldlifeexpectancy.com/ world-health-rankings (accessed 18 May 2017).

21. Ministry of Health Government of Trinidad and Tobago. Panamerican steps chronic non-communicable disease risk factor survey: Final report. http://www.who.int/chp/steps/TrinidadAndTobago_2011_ STEPS_Report.pdf.

22. Prince $M$, Ferri CP, Acosta $D$, et al. The protocols for the $10 / 66$ dementia research group population-based research programme. BMC Public Health 2007;7:165.

23. Llibre Rodriguez JJ, Ferri CP, Acosta D, et al. Prevalence of dementia in Latin America, India, and China: a population-based crosssectional survey. Lancet 2008;372:464-74.

24. Prince M, Bryce R, Albanese E, et al. The global prevalence of dementia: a systematic review and metaanalysis. Alzheimers Dement 2013;9:63-75.

25. Langa KM, Larson EB, Crimmins EM, et al. A comparison of the prevalence of dementia in the United States in 2000 and 2012. JAMA Intern Med 2017;177:51-8.

26. Wu YT, Beiser AS, Breteler MMB, et al. The changing prevalence and incidence of dementia over time - current evidence. Nat Rev Neurol 2017;13:327-39.

27. Global Age Watch Index 2013, Purpose, methodology and results. www.helpage.org (accessed 18 May 2017). 
28. https://idf.org/our-network/regions-members/north-america and Caribbean/welcome.html (accessed 18 May 2017).

29. Matthews FE, Stephan BC, Robinson L, et al. A two decade dementia incidence comparison from the Cognitive Function and Ageing Studies I and II. Nat Commun 2016;7:11398.

30. Solomon A, Kivipelto M, Wolozin B, et al. Midlife serum cholesterol and increased risk of Alzheimer's and vascular dementia three decades later. Dement Geriatr Cogn Disord 2009;28:75-80.

31. Whitmer RA, Sidney S, Selby J, et al. Midlife cardiovascular risk factors and risk of dementia in late life. Neurology 2005;64:277-81.

32. Birns J, Kalra L. Cognitive function and hypertension. J Hum Hypertens 2009;23:86-96.

33. Kennelly SP, Lawlor BA, Kenny RA. Blood pressure and the risk for dementia: a double edged sword. Ageing Res Rev 2009;8:61-70.

34. Corrada M, Hayden KM, Bullain SS, et al. Age of onset of hypertension and risk of dementia in the oldest-old: The 901 study. Alzheimers \& Dementia 2014;10(4Suppl):501.
35. World Health Organization. Dementia: A public health priority 2012. https://extranet.who.int/agefriendlyworld/wp-content/uploads/2014/ 06/WHO- Dementia-English.pdf (accessed 10 May 2017).

36. Dementia in the Americas: Current and Future Cost and the Prevalence of Alzheimer's Disease and other Dementias. Alzheimer's Disease International. https://www.alz.co.uk/sites/ default/files/pdfs/dementia-in-the-americas-ENGLISH.pdf (accessed 18 May 2017).

37. Noguchi-Shinohara M, Yuki S, Dohmoto C, et al. Differences in the prevalence of dementia and mild cognitive impairment and cognitive function between early and delayed responders in a community-based study of the elderly. J Alzheimer's Dis 2013;37:691-8.

38. Prince MJ. The 10/66 dementia research group -10 years on Indian. J Psychiatry 2009;51(Suppl 1):S8-15.

39. Judge GG, Griffiths WE, Hill RC, ed. The Theory and Practice of Econometrics. 2nd. New York: Wiley, 1985. 